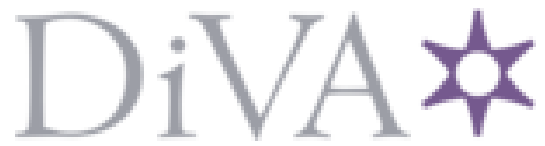

http://www.diva-portal.org

This is the published version of a paper published in interactions.

Citation for the original published paper (version of record):

Balaam, M., Hansen, L K. (2018)

Women's health at CHI.

interactions, 25(1)

https://doi.org/10.1145/3169797

Access to the published version may require subscription.

N.B. When citing this work, cite the original published paper.

Permanent link to this version:

http://urn.kb.se/resolve?urn=urn:nbn:se:kth:diva-222521 


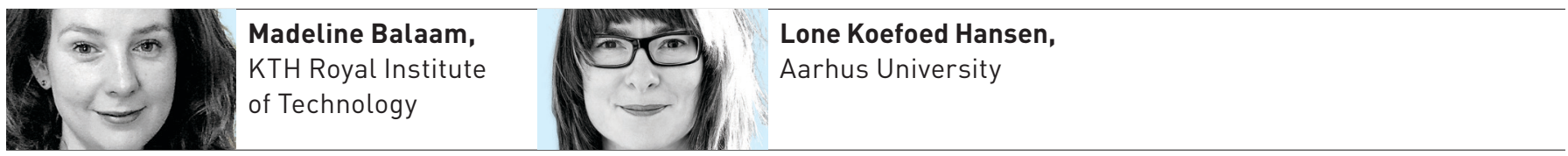

\section{Women’s Health @ CHI}

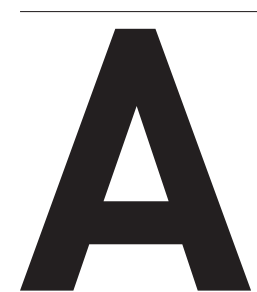

t CHI 2017 we

ran a workshop to reimagine how technology intersects with women's health. We brought together designers, engineers, programmers, and experts in women's health over two days in an attempt to radically re-engineer the ways in which women receive healthcare. We made use of the excellent public maker spaces in Denver's Central Public library (https://www.denverlibrary. org/idealab) to build exemplary digital interactions that demonstrate the kinds of innovations we consider necessary to improve women's health on a global scale.

Throughout our two-day event, 25 participants generated many responses: developing a digital inclusive parenting campaign, hacking vibrators, experimenting with personal visualizations of menstrual cycles, discussing technologies for menopausal women, talking about what it means to be inclusive and exclusive of gender norms, and experimenting with DIYbio protocols to explore feminist science. We had put the needs and hopes for people who identify as women front and center for two days, and through this we realized just how rare it is to do so without having to explain why.

The workshop felt timely and urgent for many participants. President Trump had been inaugurated several months earlier, and one of his first acts was to cut funding to international organizations that promote women's health if they also offer access to abortion services, or even provide advice and information to women about these services. The NGOs affected by this policy have highlighted how such an act will decrease marginalized people's access to vital sexual and reproductive health services, and increase illegal abortions and ultimately mortality. Times are hard, but there are ripples of action being taken globally, such as the \#Repealthe8th campaign using humor to draw attention to the lack of abortion services in Ireland; the Pussy Hats project, which creates a visual symbol for those advocating for women's rights in a context of everyday sexual harassment; and the "reproductive justice" hackathon that took place in March last year. At an institutional level, some heads of state readily identify as feminist (hello, Sweden and Canada), and some countries have begun to officially recognize non-binary genders in passports. Even CHI 2017 had a few gender-neutral restrooms.

However, it's also not easy to do research in an area when the majority of people in the field think it is only relevant for a minority. We are acutely attuned to how the current political and social climate impacts our work. We notice how even the act of naming our workshop Hacking Women's Health can be seen as taking an outsider or oppositional stance, even if half the world's population identify as female. We have been innovating digital technologies for

\section{By limiting the organizations we can work with during SIGCHI events, do we limit the impact of the community?}

women's health over a number of years, from FeedFinder (supporting women in breastfeeding in public), to hacking breastpumps, to Labella (an app to develop awareness of intimate anatomy). By hosting this workshop at CHI we hoped we could not only contribute to these global ripples of action and resistance, but also increase the community, profile, and voice of researchers working in this area. Because, right now-we'll be honest - it can be difficult working on this topic. Reviewers have told us that our research is not science, is not ethical, is unseemly, is not feminist, or is too feminist. In contrast to research we have undertaken in other areas, it seems to us that this work is often held to a higher standard; more is required of us to prove it is worth publishing, or that it is even research at all.

This has been our experience of trying to investigate women's health at SIGCHI venues since running the Motherhood and HCI workshop at CHI 2013. There we had conversations with other attendees who wanted to spend their research time contributing to agendas around women's health and motherhood, but couldn't quite find the right way to persuade their research groups and organizations to allow them to engage with such topics. Or who had to invest in long discussions as to why a topic that "only" affected women was worthy of more than a smile. Somehow thinking about women's health and the female body is considered daring and brave, but designing for the intersection between technology and the female body is taboo. It sits on the fringe of CHI, though it should be at the center. There are untold opportunities for technical and interactional innovations focused on women's health. Those thinking about on-body 
and wearable computing might find their "killer apps" exactly within this space. There are rich opportunities for designing for advocacy and activism, alongside extremely complex and sensitive settings that are entirely unchartered within the community. We need to find ways of making this type of research mainstream, because the potential for HCI to create positive impacts for women globally is great.

But why is it so hard to legitimize work in this area? Well, this year we think we're starting to understand. We are passionate about women's health and women's access to healthcare services, and angry that this access is being limited and that women's lives are at risk as a result. We're angry enough that we wanted to use our Hacking Women's Health workshop as an opportunity not only to talk about our research, exchange ideas, and be creative, but also to generate impact outside our community. We initially started organizing our workshop around activities that would generate crafts that we could sell at the conference to generate small donations to Planned Parenthood, an essential women's and sexual health NGO. However, we were unable to do this since fundraising for any particular cause is not permitted at SIGCHI conferences.

In the past couple of years, the $\mathrm{CHI}$ conference has run CHI4Good days of service. The thrust of these days has not been to raise funds, but rather to offer time and support (technical, design, research, and otherwise) to selected charities as part of the conference. This is a great way of ensuring meaningful impact for the wider global community. But we wondered why it was that CHI could support some charities in these ways and not others. The answer we received was that charities had applied and been chosen in advance of the conference, and that organizations would not have been selected for CHI4Good if considered to be of a "polarizing political nature." So is Planned Parenthood too politically polarizing? Can $\mathrm{CHI}$ and its attendees contribute to charities and organizations only as long as they are

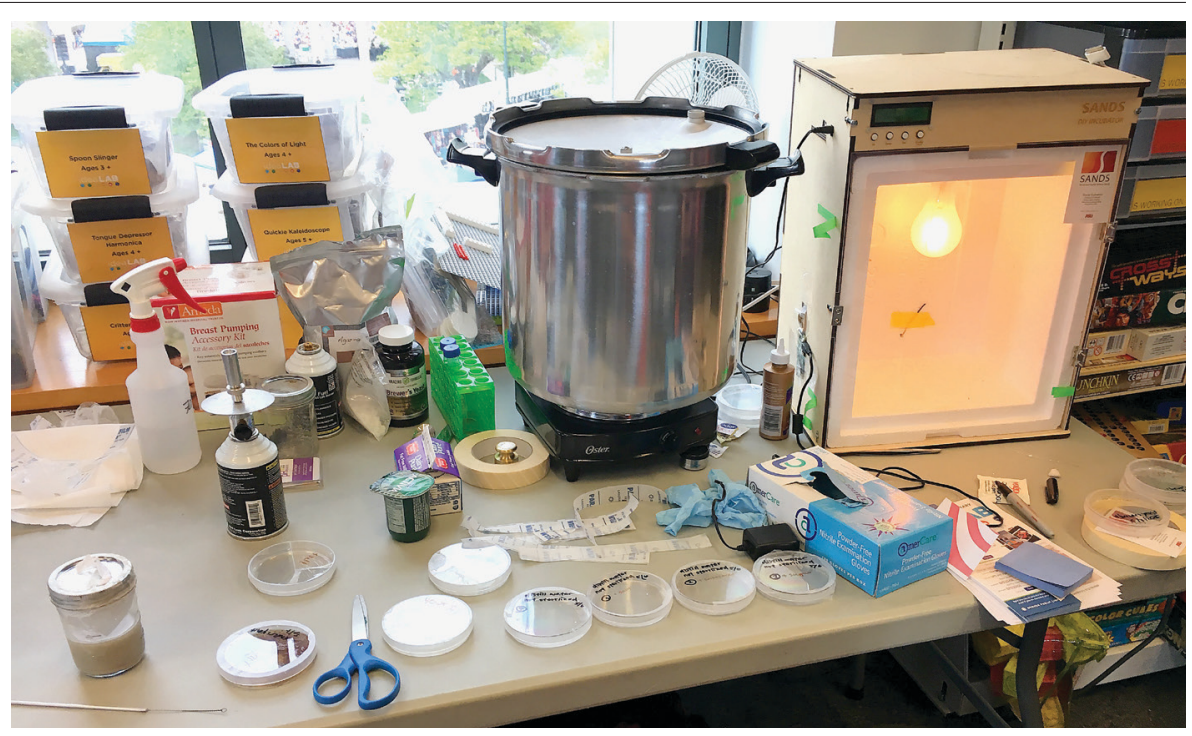

A portable bio lab that one group used to practice sterilization and culture yeast while discussing Internet memes, zine culture, and other feminist tactics.

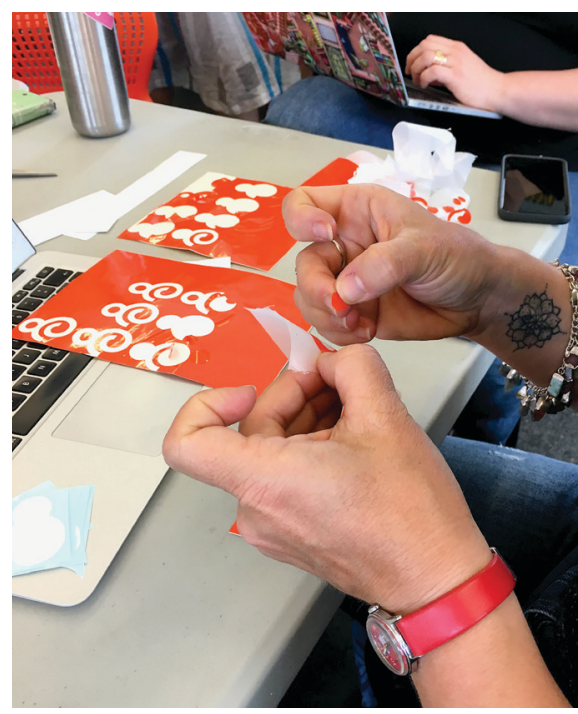

In the "inclusive parenting" group, participants are working on the (digital) campaign material that came as a result of the discussions, beginning with breastfeeding.

considered not too divisive? Animals, children, cycling, yes? Reproductive rights and providing healthcare to women with limited recourse to funds, no? By limiting the organizations we can work with during SIGCHI events, do we limit the impact of the community and potentially marginalize the organizations and charities that need support, in favor of those that are potentially perceived to be more agreeable? By drawing these lines, what falls outside? Increasingly it seems the radical or controversial, and in this current

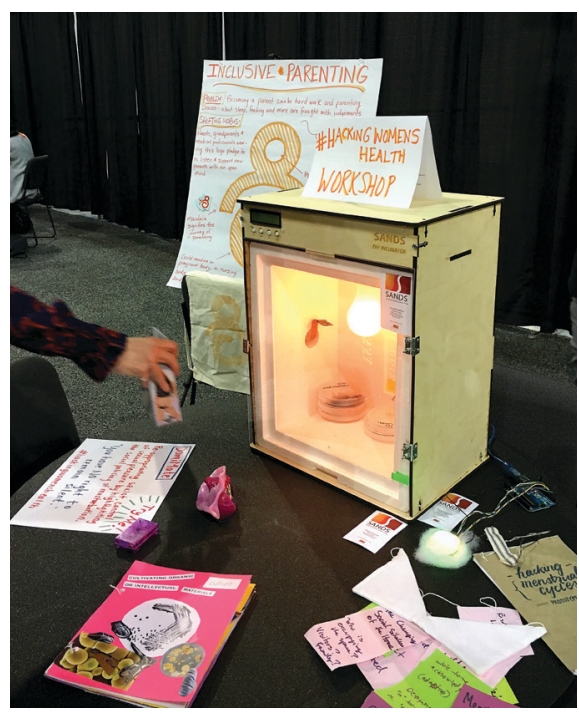

The workshop "hacked" the exhibition space at the opening reception by installing the results from the workshop on a table next to an ice-cream stand.

climate basic women's health services and information, at the cost of further stigmatizing and delegitimizing women's health as an area of inquiry.

(1) Madeline Balaam designs interactions for women's health, digital health, and well-being $\rightarrow$ balaamakth.se

(1) Lone Koefoed Hansen works with feminist design, critical computing, and participatory IT. $\rightarrow$ koefoeddacavi.au.dk

Note: Catherine D'Ignazio of Emerson College, Stacey Kuznetsov of Arizona State University, and Emma Simpson of Newcastle University also contributed to this blog post. 